Article

\title{
Fixed Point Theorems through Modified $\omega$-Distance and Application to Nontrivial Equations
}

\author{
Tariq Qawasmeh ${ }^{1}$, Abdalla Tallafha ${ }^{1}$ and Wasfi Shatanawi ${ }^{2,3, *}$ \\ 1 Department of mathematics, School of Science, University of Jordan, Amman 11942, Jordan; \\ jorqaw@yahoo.com or tar9160251@fgs.ju.edu.jo (T.Q.); a.tallafha@ju.edu.jo (A.T.) \\ 2 Department of Mathematics and general courses, Prince Sultan University, Riyadh 11586, Saudi Arabia \\ 3 Department of Medical Research, China Medical University Hospital, China Medical University, \\ Taichung 40402, Taiwan \\ * Correspondence: wshatanawi@psu.edu.sa or wshatanawi@yahoo.com
}

Received: 27 April 2019; Accepted: 5 May 2019; Published: 8 May 2019

\begin{abstract}
In this manuscript, we utilize the concept of modified $\omega$-distance mapping, which was introduced by Alegre and Marin [Alegre, C.; Marin, J. Modified $\omega$-distance on quasi metric spaces and fixed point theorems on complete quasi metric spaces. Topol. Appl. 2016, 203, 120-129] in 2016 to introduce the notions of $(\omega, \varphi)$-Suzuki contraction and generalized $(\omega, \varphi)$-Suzuki contraction. We employ these notions to prove some fixed point results. Moreover, we introduce an example to show the novelty of our results. Furthermore, we introduce some applications for our results.
\end{abstract}

Keywords: quasi metric space; Suzuki contractions; fixed point theorems; modified $\omega$-distance; almost perfect functions

\section{Introduction and Preliminaries}

Constructing new contractions and formulating new fixed point theorems are very important subjects in mathematics since active researchers employ the existence and uniqueness of the fixed point to solve some integral equations, differential equations, etc.

Banach was the first pioneer mathematician who constructed and formulated the first fixed point theorem, which was called after him as the Banach contraction principle [1].

Suzuki [2] introduced a new contraction and generalized the Banach contraction principle.

In the rest of this paper, the letter $d$ refers to a metric on a set $B$ and $f_{1}$ refers to self-mappings on $B$.

One of the important contractions is the Kannan contraction [3]:

$$
d\left(f_{1} l_{1}, f_{1} l_{2}\right) \leq \alpha\left[d\left(l_{1}, f_{1} l_{1}\right)+d\left(l_{2}, f_{1} l_{2}\right)\right] \text { for all } l_{1}, l_{2} \in B
$$

where $\alpha \in\left[0, \frac{1}{2}\right)$.

Moreover, Kannan proved that if $f_{1}$ satisfies Kannan contraction, then $f_{1}$ has a unique fixed point.

In 1931, Wilson [4] generalized the notion of metric spaces to a new notion called quasi metric spaces.

Definition 1. We call $q: B \times B \rightarrow[0, \infty)$ a quasi metric if $q$ satisfies:

(i) $\quad q\left(l_{1}, l_{2}\right)=0 \Longleftrightarrow l_{1}=l_{2}$

and:

(ii) $q\left(l_{1}, l_{2}\right) \leq q\left(l_{1}, l_{3}\right)+q\left(l_{3}, l_{2}\right)$ for all $l_{1}, l_{2}, l_{3} \in B$.

$(B, q)$ is called a quasi metric space. 
From now on, by $(B, q)$, we mean a quasi metric space.

Defining $q_{m}: B \times B \rightarrow[0,+\infty)$ via

$$
q_{m}\left(l_{1}, l_{2}\right)=\max \left\{q\left(l_{1}, l_{2}\right), q\left(l_{2}, l_{1}\right)\right\},
$$

we generate a metric on $B$.

Recall the following definitions.

Definition 2. [5,6] The sequence $\left(l_{t}\right)$ converges to $l \in B$ if $\lim _{t \rightarrow \infty} q\left(l_{t}, l\right)=\lim _{n \rightarrow \infty} q\left(l, l_{t}\right)=0$.

Definition 3. [6] Let $\left(l_{t}\right)$ be a sequence in $(B, q)$. Then, we say that:

(i) $\quad\left(l_{t}\right)$ is left-Cauchy if for any $\varepsilon>0$, there exists $n_{0} \in \mathbb{N}$ such that $q\left(l_{t}, l_{m}\right)<\varepsilon \forall t \geq m>n_{0}$.

(ii) $\left(l_{t}\right)$ is right-Cauchy if for any $\varepsilon>0$, there exists $n_{0} \in \mathbb{N}$ such that $q\left(l_{t}, l_{m}\right)<\varepsilon \forall m \geq t>n_{0}$.

Definition 4. [5,6] We say that $\left(l_{t}\right)$ is Cauchy if for any $\varepsilon>0$, there exists $n_{0} \in \mathbb{N}$ such that $q\left(l_{t}, l_{m}\right) \leq \epsilon \forall$ $t, m>n_{0}$.

We note that $\left(l_{t}\right)$ in $(B, q)$ is Cauchy if and only if $\left(l_{t}\right)$ is right and left Cauchy.

Definition 5. [5,6] We say that $(B, q)$ is complete if every Cauchy sequence in $B$ is convergent.

For some theorems in quasi-metric space, see [5-9].

Alegre and Marin [10] introduced the concept of modified $\omega$-distance mappings on $(B, d)$.

Definition 6. [10] A modified $\omega$-distance (shortened as mw-distance) on $(B, q)$ is a function $p: B \times B \rightarrow$ $[0, \infty)$, which satisfies:

(W1) $\quad p\left(l_{1}, l_{2}\right) \leq p\left(l_{1}, l_{3}\right)+p\left(l_{3}, l_{2}\right)$ for all $l_{1}, l_{2}, l_{3} \in B$;

(W2) $\quad p(l,):. B \rightarrow[0, \infty)$ is lower semi-continuous for all $l \in B$; and

(mW3) for each $\varepsilon>0$, there exist $v>0$ such that if $p\left(l_{1}, l_{2}\right) \leq v$ and $p\left(l_{2}, l_{3}\right) \leq v$, then $q\left(l_{1}, l_{3}\right) \leq \epsilon$ for all $l_{1}, l_{2}, l_{3} \in B$.

Definition 7. [10] We call an mw-distance function a $p$ strong $m \omega$-distance if $p$ is lower semi-continuous on its second coordinate.

Remark 1. [10] If $q$ is a quasi metric on $B$, then $q$ is mw-distance.

Lemma 1. [11] Let $\left(\alpha_{t}\right),\left(\beta_{t}\right)$ be two sequences of nonnegative real numbers converging to zero. Assume that $p$ is $m \omega$-distance. Then, we have the following:

(i) If $p\left(l_{t}, l_{m}\right) \leq \alpha_{t}$ for any $t, m \in \mathbb{N}$ with $m \geq t$, then $\left(l_{t}\right)$ is right Cauchy in $(B, q)$.

(ii) If $p\left(l_{t}, l_{m}\right) \leq \beta_{m}$ for any $t, m \in \mathbb{N}$ with $t \geq m$, then $\left(l_{t}\right)$ is left Cauchy in $(B, q)$.

Remark 2. [11] The above lemma implies that if $\lim _{m, t \rightarrow \infty} p\left(l_{t}, l_{m}\right)=0$, then $\left(l_{t}\right)$ is Cauchy in $(B, q)$.

For some works on $\omega$-distance, we ask the readers to see [11-13].

Abodayeh et al. [14] generalized the definition of altering the distance function [15] to the concept of the almost perfect function.

Definition 8. We call a non-decreasing function $\varphi:[0, \infty) \rightarrow[0, \infty)$ almost perfect if $\varphi$ satisfies:

(i) $\varphi(l)=0$ if and only if $l=0$.

(ii) If $\left(l_{t}\right)$ is a sequence in $[0, \infty)$ such that $\lim _{t \rightarrow \infty} \varphi\left(l_{t}\right)=0$, then $\lim _{t \rightarrow \infty} l_{t}=0$. 


\section{Main Results}

We begin our work with the following definition:

Definition 9. Let $\varphi: \mathbb{R}^{+} \rightarrow \mathbb{R}^{+}$be an almost perfect function and $p$ be modified $\omega$-distance on $B$. We say that $p$ is bounded with respect to $\varphi$ if there exists an integer $A>0$ such that:

$$
\varphi p(l, e) \leq A \text { for all } l, e \in B
$$

Definition 10. Equip $(B, q)$ with an mw-distance mapping $p$. Then, we call that $f_{1}: B \rightarrow B$ an $(\omega, \varphi)$-Suzuki contraction if there are an almost perfect function $\varphi$ and a constant $k \in[0,1)$ such that for all $l, e \in X$ and $t \in \mathbb{N}$, we have:

$$
(1-k) p\left(l, f_{1}^{t} l\right) \leq p(l, e) \Longrightarrow \varphi p\left(f_{1} l, f_{1} e\right) \leq k \varphi p(l, e)
$$

and:

$$
(1-k) p\left(f_{1}{ }^{t} l, l\right) \leq p(e, l) \Longrightarrow \varphi p\left(f_{1} e, f_{1} l\right) \leq k \varphi p(e, l)
$$

Now, we introduce and prove our first result.

Theorem 1. Equip $(B, q)$ with an mw-distance mapping $p$. Let $p$ be bounded with respect to the almost perfect function $\varphi$ and $f_{1}$ be an $(\omega, \varphi)$-Suzuki contraction mapping. Suppose that:

(i) $f_{1}$ is continuous,

or

(ii) if $u^{*} \in B$ and $u^{*} \neq f_{1} u^{*}$, then:

$$
\inf \left\{p\left(e, u^{*}\right)+p\left(f_{1} e, u^{*}\right): e \in B\right\}>0 \text {. }
$$

Then, $f_{1}$ has a unique fixed point in $B$.

Proof. By starting with $l_{0} \in B$, we produce a sequence $\left(l_{t}\right)$ in $B$ inductively by putting $l_{t+1}=f_{1} l_{t}$ for all $t \in \mathbb{N} \cup\{0\}$. Given $m, t \in \mathbb{N} \cup\{0\}$ with $m>t$, then $m=t+s$ for some $s \in \mathbb{N}$. From the definition, we have:

$$
\begin{aligned}
(1-k) p\left(l_{t-1}, l_{m-1}\right) & =(1-k) p\left(l_{t-1}, l_{t+s-1}\right) \\
& \leq p\left(l_{t-1}, l_{t+s-1}\right) .
\end{aligned}
$$

Therefore, we get that:

$$
\begin{aligned}
\varphi p\left(l_{t}, l_{m}\right) & =\varphi p\left(f_{1} l_{t-1}, f_{1}{ }^{s} l_{t-1}\right) \\
& =\varphi p\left(f_{1} l_{t-1}, f_{1} l_{t+s-1}\right) \\
& \leq k \varphi p\left(l_{t-1}, l_{t+s-1}\right) .
\end{aligned}
$$

Repeating (2) t-times, we get that:

$$
\varphi p\left(l_{t}, l_{m}\right) \leq k^{t} \varphi p\left(l_{0}, l_{s}\right) .
$$

Since $(B, p)$ is bounded with respect to $\varphi$, then we have:

$$
\varphi p\left(l_{t}, l_{m}\right) \leq k^{t} A \text { for some integer } A>0 .
$$

By letting $t, m \rightarrow \infty$, we get that:

$$
\lim _{t, m \rightarrow \infty} \varphi p\left(l_{t}, l_{m}\right)=0 .
$$


By the definition of $\varphi$, we get that:

$$
\lim _{t, m \rightarrow \infty} p\left(l_{t}, l_{m}\right)=0 .
$$

Since $m>t$, Lemma 1 implies that $\left(l_{t}\right)$ is right Cauchy. Now, suppose that $t, m \in \mathbb{N} \cup\{0\}$ with $t>m$. Then, $t=m+q$ for some $q \in \mathbb{N}$. We note that:

$$
(1-k) p\left(l_{t-1}, l_{m-1}\right) \quad \leq p\left(l_{m+q-1}, l_{m-1}\right) .
$$

Therefore, we get that:

$$
\begin{gathered}
\varphi p\left(l_{t}, l_{m}\right) \quad=\varphi p\left(f_{1} l_{t-1}, f_{1} x_{m-1}\right) \\
\leq \cdots \leq k^{m} \varphi p\left(l_{q}, l_{0}\right)
\end{gathered}
$$

Since $(B, p)$ is bounded with respect to $\varphi$, we get that:

$$
\varphi p\left(l_{n}, l_{m}\right) \leq k^{m} A \text { for some integer } A>0 .
$$

By letting $t, m \rightarrow \infty$, we have:

$$
\lim _{t, m \rightarrow \infty} \varphi p\left(l_{n}, l_{m}\right)=0
$$

Therefore,

$$
\lim _{t, m \rightarrow \infty} p\left(l_{t}, l_{m}\right)=0 .
$$

Since $t>m$, Lemma 1 implies that $\left(l_{t}\right)$ is left Cauchy. Therefore, we deduce that $\left(l_{t}\right)$ is Cauchy. The completeness of $(B, q)$ implies that there exists an element $l^{*} \in B$ such that $l_{t} \rightarrow l^{*}$. If $f_{1}$ is continuous, then $l_{t+1}=f_{1} l_{t}$ converges to $f_{1} l^{*}$. The uniqueness of the limit ensures that $f_{1} l^{*}=l^{*}$. Let $\epsilon>0$. Since $\lim _{t, m \rightarrow \infty} p\left(l_{t}, l_{m}\right)=0$, we choose $k_{0} \in \mathbb{N}$ such that $p\left(l_{t}, l_{m}\right) \leq \frac{\epsilon}{2}$ for all $l, m \geq k_{0}$. The lower semi continuity of $p$ implies that:

$$
p\left(l_{t}, l^{*}\right) \leq \lim _{j \rightarrow \infty} \inf p\left(l_{t}, l_{j}\right) \leq \frac{\epsilon}{2} \text { for all } n \geq k_{0} .
$$

Assume that $l^{*} \neq f_{1} l^{*}$. Then, by (1), we have:

$$
\begin{aligned}
\inf \left\{p\left(e, l^{*}\right)+p\left(f_{1} e, l^{*}\right)\right. & : e \in B\} \leq \inf \left\{p\left(l_{t}, l^{*}\right)+p\left(f_{1} l_{t}, l^{*}\right): t \in \mathbb{N}\right\} \\
& =\inf \left\{p\left(l_{t}, l^{*}\right)+p\left(l_{t+1}, l^{*}\right): t \in \mathbb{N}\right\} \leq \epsilon,
\end{aligned}
$$

a contradiction. Therefore, $l^{*}=f_{1} l^{*}$. Now, assume that $z^{*} \in B$ is a fixed point of $f_{1}$. Therefore:

$$
(1-k) p\left(z^{*}, f_{1}{ }^{t} z^{*}\right)=(1-k) p\left(z^{*}, z^{*}\right) \leq p\left(z^{*}, z^{*}\right) .
$$

Thus,

$$
\varphi p\left(z^{*}, z^{*}\right)=\varphi p\left(f_{1} z^{*}, f_{1} z^{*}\right) \leq k \varphi p\left(z^{*}, z^{*}\right) .
$$

Since $k<1$ and $\varphi$ is an almost perfect function, we conclude that $p\left(z^{*}, z^{*}\right)=0$. Assume that there exists $v^{*} \in B$ such that $v^{*}=f_{1} v^{*}$. Since $p\left(z^{*}, z^{*}\right)=0$, we have:

$$
(1-k) p\left(z^{*}, f_{1}^{t} z^{*}\right)=(1-k) p\left(z^{*}, z^{*}\right) \leq p\left(z^{*}, v^{*}\right) .
$$

Therefore,

$$
\varphi p\left(z^{*}, v^{*}\right)=\varphi p\left(f_{1} z^{*}, f_{1} v^{*}\right) \leq k \varphi p\left(z^{*}, v^{*}\right) .
$$


Thus, we have $\varphi p\left(z^{*}, v^{*}\right)=0$, and so, $p\left(z^{*}, v^{*}\right)=0$. Hence, by (mW3), we have $q\left(z^{*}, v^{*}\right)=0$. Thus, $v^{*}=z^{*}$. Therefore, the fixed point of $f_{1}$ is unique.

Corollary 1. Equip $(B, q)$ with an $m \omega$-distance mapping $p$. Assume $p$ is bounded with respect to $\varphi$. Assume for all $e, l \in B$, we have:

$$
\varphi p\left(f_{1} e, f_{1} l\right) \leq k \varphi(p(e, l)) \text {, where } k \in[0,1) \text {. }
$$

Furthermore, assume that:

(i) $f_{1}$ is continuous,

or

(ii) if $u^{*} \in B$ and $u^{*} \neq f_{1} u^{*}$, then:

$$
\inf \left\{p\left(e, u^{*}\right)+p\left(f_{1} e, u^{*}\right): e \in B\right\}>0 .
$$

Then, $f_{1}$ has a unique fixed point in $B$.

By taking the almost perfect function $\varphi$ in Corollary 1 as follows:

$\varphi(e)=e$, we get the following result:

Corollary 2. Equip $(B, q)$ with an $m \omega$-distance mapping $p$. Assume there exists $A>0$ such that $p(e, l) \leq A$ for all e, $l \in B$. Furthermore, assume that there exists $k \in[0,1)$ such that for all e, $l \in B$, we have:

$$
p\left(f_{1} e, f_{1} l\right) \leq k p(e, l), \text { where } k \in[0,1) .
$$

Furthermore, assume that:

(i) $f_{1}$ is continuous,

or

(ii) if $u^{*} \in B$ and $u^{*} \neq f_{1} u^{*}$, then:

$$
\inf \left\{p\left(e, u^{*}\right)+p\left(f_{1} e, u^{*}\right): e \in B\right\}>0 .
$$

Then, $f_{1}$ has a unique fixed point in $B$.

Example 1. Let $B=\{0,1,2, \cdots, n\}$, where $n \in \mathbb{N}$. Define $p, q: B \times B \rightarrow[0,+\infty)$ as follows:

$$
q(e, l)= \begin{cases}0 & \text { if } e=l \\ 3 e+l & \text { if } e \neq l\end{cases}
$$

and:

$$
p(e, l)= \begin{cases}0 & \text { if } e=l \\ \frac{1}{2}(3 e+l) & \text { if } e \neq l\end{cases}
$$

Furthermore, define $f_{1}: B \rightarrow B$ by:

$$
f_{1} e= \begin{cases}0 & \text { if } e=0,1 \\ 1 & \text { if } e=2,3, \cdots, n,\end{cases}
$$

and $\varphi: \mathbb{R}^{+} \rightarrow \mathbb{R}^{+}$by:

$$
\varphi(l)= \begin{cases}3^{l}-1 & \text { if } l \in[0, n] \\ 3^{l} & \text { if } l>n\end{cases}
$$


Then,

1. $\varphi$ is an almost perfect function.

2. $\quad p$ is an $m \omega$-distance function on $q$.

3. $q$ is a quasi metric on $B$.

4. $(B, q)$ is complete.

5. $f_{1}$ satisfies $(\omega, \varphi)$-Suzuki contraction with $k=\frac{1}{\sqrt{3}}$, i.e., $\forall e, l \in B, j \in \mathbb{N}$, we have:

$$
\left(1-\frac{1}{\sqrt{3}}\right) p\left(e, f_{1}^{j} e\right) \leq p(e, l) \Longrightarrow \varphi p\left(f_{1} e, f_{1} l\right) \leq k \varphi p(e, l),
$$

and:

$$
\left(1-\frac{1}{\sqrt{3}}\right) p\left(f_{1}^{j} e, e\right) \leq p(l, e) \Longrightarrow \varphi p\left(f_{1} l, f_{1} e\right) \leq k \varphi p(l, e)
$$

Proof. The proofs of (1), (2), and (3) are obvious. To show that $q$ is complete, let $\left(l_{t}\right)$ be a Cauchy sequence in $B$. Then, for each $t, m \in \mathbb{N}$, we have:

$$
\lim _{m, t \rightarrow \infty} q\left(l_{t}, l_{m}\right)=0
$$

Therefore, we deduce that $l_{t}=l_{m}$ for all $t, m \in\{0,1,2, \cdots\}$, but possible for finitely many. Thus, $\left(l_{t}\right)$ converges in $B$. Hence, $(B, q)$ is complete. To prove (5), given $e, l \in B$, we divide our proof into the following cases: Case (1): $e=0$. Here, we have:

$$
\left(1-\frac{1}{\sqrt{3}}\right) p(0,0)=\left(1-\frac{1}{\sqrt{3}}\right) p\left(e, f_{1}^{j} e\right) \leq p(0, l) \text { where } l=0,1, \cdots, n
$$

If $l \in\{0,1\}$, then:

$$
\varphi p\left(f_{1} 0, f_{1} l\right)=\varphi p(0,0)=0 \leq\left(\frac{1}{\sqrt{3}}\right) \varphi p(0, l)
$$

If $l \in\{2,3, \cdots, n\}$, then:

$$
\varphi p\left(f_{1} 0, f_{1} l\right)=\varphi p(0,1)=\varphi\left(\frac{1}{2}\right)=3^{\frac{1}{2}}-1
$$

Therefore,

$$
\begin{gathered}
\varphi p(0, l)=\varphi\left(\frac{l}{2}\right)=3^{\frac{l}{2}}-1 . \\
\varphi p\left(f_{1} 0, f_{1} l\right)=3^{\frac{1}{2}}-1 \leq\left(\frac{1}{\sqrt{3}}\right)\left(3^{\frac{l}{2}}-1\right) .
\end{gathered}
$$

Case (2): $e=1$. Here:

$$
\left(1-\frac{1}{\sqrt{3}}\right) p(e, 0)=\left(1-\frac{1}{\sqrt{3}}\right) p\left(1, f_{1} 1\right) \leq p(1, l) \text { where } l=0,2,3, \cdots, n
$$

If $l=0$, then we have $\varphi p(f 1, f l)=0$. Therefore,

$$
\varphi p(f 1, f l)=0 \leq\left(\frac{1}{\sqrt{3}}\right)\left(3^{\frac{3}{2}}-1\right) .
$$

If $l=2,3, \cdots, n$, then:

$$
\varphi p\left(f_{1} 1, f_{1} l\right)=\varphi p(0,1)=\varphi\left(\frac{1}{2}\right)=3^{\frac{1}{2}}-1 .
$$


Now,

$$
\varphi p(1, l)=\varphi\left(\frac{3+l}{2}\right)=3^{\frac{3+l}{2}}-1
$$

Thus,

$$
\varphi p\left(f_{1} 1, f_{1} l\right)=3^{\frac{1}{2}}-1 \leq\left(\frac{1}{\sqrt{3}}\right) \varphi\left(\frac{3+l}{2}\right)=\left(\frac{1}{\sqrt{3}}\right)\left(3^{\frac{3+l}{2}}-1\right) .
$$

Case (3): $e \in\{2,3, \cdots, n\}$. Here,

$$
\left(1-\frac{1}{\sqrt{3}}\right) p(e, 1)=\left(1-\frac{1}{\sqrt{3}}\right) p\left(e, f_{1} e\right) \leq p(e, l) \text { where } l=1,2, \cdots, n
$$

If $l=1$, then:

$$
\begin{gathered}
\varphi p(f e, f 1)=\varphi p(1,0)=\varphi\left(\frac{3}{2}\right)=3^{\frac{3}{2}}-1 . \\
\varphi p(e, 1)=\varphi\left(\frac{3 e+1}{2}\right)= \begin{cases}3^{\frac{7}{2}}-1 & \text { if } e=2 \\
3^{\frac{3 e+1}{2}} & \text { if } 3 \leq e \leq n .\end{cases} \\
\varphi p(f e, f 1)=3^{\frac{3}{2}}-1 \leq\left(\frac{1}{\sqrt{3}}\right) \varphi p(e, 1) .
\end{gathered}
$$

If $l \in\{2,3, \cdots, n\}, e \in\{2,3, \cdots, n\}$ and $e \neq l$, then:

$$
\begin{gathered}
\varphi p\left(f_{1} e, f_{1} l\right)=\varphi p(1,1)=\varphi(0)=0 . \\
\varphi p(e, l)=\varphi\left(\frac{3 e+l}{2}\right)= \begin{cases}3^{\frac{3 e+l}{2}}-1 & \text { if } 3 e+l \leq 2 n \\
3^{\frac{3 e+l}{2}} & \text { if } 3 e+l>2 n .\end{cases}
\end{gathered}
$$

Similarly, we can show that:

$$
\left(1-\frac{1}{\sqrt{3}}\right) p\left(f^{t} e, e\right) \leq p(l, e) \Longrightarrow \varphi p(f l, f e) \leq k \varphi p(l, e) .
$$

Hence, $f_{1}$ satisfies $(\omega, \varphi)$-Suzuki contraction. Therefore, $f_{1}$ has a unique fixed point.

Next, we introduce the definition of a generalized $(\omega, \varphi)$-Suzuki contraction.

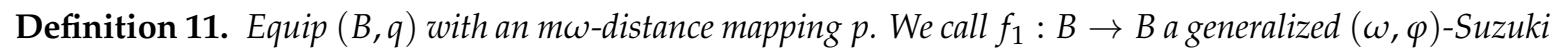
contraction if there exists an ultra distance function $\varphi$ and a constant $k \in[0,1)$ such that for all $e, l \in B, j \in \mathbb{N}$, we have:

$$
(1-k) p\left(e, f_{1}^{j} e\right) \leq p(e, l) \Longrightarrow \varphi p\left(f_{1} e, f_{1} l\right) \leq k \max \left\{\varphi p\left(e, f_{1} e\right), \varphi p\left(l, f_{1} l\right)\right\},
$$

and:

$$
(1-k) p\left(f_{1}^{j} e, e\right) \leq p(l, e) \Longrightarrow \varphi p\left(f_{1} l, f_{1} e\right) \leq k \max \left\{\varphi p\left(e, f_{1} e\right), \varphi p\left(l, f_{1} l\right)\right\} .
$$

We introduce and prove the second result:

Theorem 2. Equip $(X, q)$ with an mw-distance mapping $p$. Assume that $p$ is bounded with respect to the almost perfect function $\varphi$. Assume that $f_{1}$ is a generalized $(\omega, \varphi)$-Suzuki contraction mapping. Furthermore, suppose that:

(i) $f_{1}$ is continuous,

or

(ii) if $u^{*} \in B$ and $u^{*} \neq f_{1} u^{*}$, then:

$$
\inf \left\{p\left(e, u^{*}\right)+p\left(f_{1} e, u^{*}\right): e \in B\right\}>0 .
$$


Then, $f_{1}$ has a unique fixed point in $B$.

Proof. Start with $l_{0} \in B$ to construct $\left(l_{n}\right)$ in $B$ inductively by putting $l_{t+1}=f_{1} l_{t}$ for all $t \in \mathbb{N} \cup\{0\}$. Given $t, m \in \mathbb{N} \cup\{0\}$ with $t<m$, let $m=t+j$ with $j \in \mathbb{N}$. We note that:

$$
\begin{aligned}
(1-k) p\left(l_{t-1}, l_{m-1}\right) & =(1-k) p\left(l_{t-1}, f_{1}^{j} l_{t-1}\right) \\
& \leq p\left(l_{t-1}, l_{m-1}\right)
\end{aligned}
$$

Since $f_{1}$ is a generalized $(\omega, \varphi)$-Suzuki contraction, we have:

$$
\begin{aligned}
\varphi p\left(l_{t}, l_{m}\right) & =\varphi p\left(f_{1} l_{t-1}, f_{1} l_{m-1}\right) \\
& \leq k \max \left\{\varphi p\left(l_{t-1}, f_{1} l_{t-1}\right), \varphi p\left(l_{m-1}, f_{1} l_{m-1}\right)\right\} \\
& \left.=k \max \left\{\varphi p\left(l_{t-1}, l_{t}\right)\right), \varphi p\left(l_{m-1}, l_{m}\right)\right\} .
\end{aligned}
$$

Now,

$$
\begin{aligned}
(1-k) p\left(l_{t-2}, l_{t-1}\right) & =(1-k) p\left(l_{t-2}, f_{1} l_{t-2}\right) \\
& \leq p\left(l_{t-2}, l_{t-1}\right) .
\end{aligned}
$$

Therefore, we get that:

$$
\begin{aligned}
\varphi p\left(l_{t-1}, l_{t}\right) & =\varphi p\left(f_{1} l_{t-2}, f_{1} l_{t-1}\right) \\
& \leq k \max \left\{\varphi p\left(l_{t-2}, f_{1} l_{t-2}\right), \varphi p\left(l_{t-1}, f_{1} l_{t-1}\right)\right\} \\
& =k \max \left\{\varphi p\left(l_{t-2}, l_{t-1}\right), \varphi p\left(l_{t-1}, l_{t}\right)\right\} .
\end{aligned}
$$

Since $k<1$, we get that:

$$
\varphi p\left(l_{t-1}, l_{t}\right) \leq k \varphi p\left(l_{t-2}, l_{t-1}\right) .
$$

Repeating (16) t-times, we get that:

$$
\varphi p\left(l_{t-1}, l_{t}\right) \leq k^{t-1} \varphi p\left(l_{0}, l_{1}\right) .
$$

Similarly, we get that that:

$$
\varphi p\left(l_{m-1}, l_{m}\right) \leq k^{m-1} \varphi p\left(l_{0}, l_{1}\right) .
$$

Using Equations (14), (17), and (18), we get:

$$
\varphi p\left(l_{t}, l_{m}\right) \leq k \max \left\{k^{t-1} \varphi p\left(l_{0}, l_{1}\right), k^{m-1} \varphi p\left(l_{0}, l_{1}\right)\right\} .
$$

Since $t<m$, we get that:

$$
\varphi p\left(l_{t}, l_{m}\right) \leq k^{t} \varphi p\left(l_{0}, l_{1}\right) .
$$

The boundedness property of $p$ with respect to $\varphi$ implies that:

$$
\varphi p\left(l_{t}, l_{m}\right) \leq k^{t} A \text { for some integer } A \geq 0 .
$$

By letting $t, m \rightarrow \infty$, we get that:

$$
\lim _{t, m \rightarrow \infty} \varphi p\left(l_{t}, l_{m}\right)=0
$$

Thus,

$$
\lim _{t, m \rightarrow \infty} p\left(l_{t}, l_{m}\right)=0 .
$$

Since $t<m$, Lemma 1 implies that $\left(l_{t}\right)$ is right Cauchy. In a similar manner, we can show that $\left(l_{t}\right)$ is left Cauchy. Hence, $\left(l_{t}\right)$ is Cauchy. The completeness of $q$ ensures that there exists $l^{*} \in B$ such that 
$\left(l_{t}\right)$ converges to $l^{*}$. If $f_{1}$ is continuous, then $\left(l_{t+1}\right)=\left(f_{1} l_{t}\right)$ converges to $f_{1} l^{*}$. The uniqueness of the limit implies that $f_{1} l^{*}=l^{*}$. Given $\varepsilon>0$. Since $\left.\lim _{t, m \rightarrow \infty} p\left(l_{t}, l_{m}\right)\right)=0$, there exists $n_{0} \in \mathbb{N}$ such that $p\left(l_{t}, l_{m}\right) \leq \frac{\epsilon}{2}$ for all $t, m \geq n_{0}$. The lower semi continuity of $p$ implies that:

$$
p\left(l_{t}, l^{*}\right) \leq \lim _{i \rightarrow \infty} \inf p\left(l_{t}, l_{i}\right) \leq \frac{\varepsilon}{2} \text { for all } t \geq n_{0} .
$$

Assume that $l^{*} \neq f_{1} l^{*}$, then by (13), we have:

$$
\begin{aligned}
\inf \left\{p\left(e, l^{*}\right)+p\left(f_{1} e, l^{*}\right)\right. & : e \in B\} \\
& \leq \inf \left\{p\left(l_{m}, l^{*}\right)+p\left(f_{1} l_{t}, l^{*}\right): t \in \mathbb{N}\right\} \\
& =\inf \left\{p\left(l_{t}, l^{*}\right)+p\left(l_{t+1}, l^{*}\right): n \in \mathbb{N}\right\} \leq \varepsilon,
\end{aligned}
$$

a contradiction. Therefore, $l^{*}=f_{1} l^{*}$. Assume $z^{*} \in B$ such that $f_{1} z^{*}=z^{*}$. First, we prove that $p\left(z^{*}, z^{*}\right)=0$. Since:

$$
(1-k) p\left(z^{*}, f_{1}^{j} z^{*}\right)=(1-k) p\left(z^{*}, z^{*}\right) \leq p\left(z^{*}, z^{*}\right),
$$

then:

$$
\varphi p\left(z^{*}, z^{*}\right)=\varphi p\left(f_{1} z^{*}, f_{1} z^{*}\right) \leq k \varphi p\left(z^{*}, z^{*}\right) .
$$

Since $k<1$ and $\varphi$ is an almost perfect function, then $p\left(z^{*}, z^{*}\right)=0$. Therefore,

$$
(1-k) p\left(z^{*}, f_{1}^{t} z^{*}\right)=(1-k) p\left(z^{*}, z^{*}\right) \leq p\left(z^{*}, l^{*}\right) .
$$

Therefore,

$$
\begin{aligned}
\varphi p\left(z^{*}, l^{*}\right) & =\varphi p\left(f_{1} z^{*}, f_{1} l^{*}\right) \\
& \leq k \max \left\{\varphi p\left(z^{*}, f_{1} z^{*}\right), \varphi p\left(l^{*}, f_{1} l^{*}\right)\right\} \\
& =k \max \left\{\varphi\left(p\left(z^{*}, z^{*}\right)\right), \varphi\left(p\left(v^{*}, v^{*}\right)\right)\right\} \\
& =0 .
\end{aligned}
$$

The definition of $\varphi$ informs us that $p\left(z^{*}, l^{*}\right)=0$. The definition of $p$ implies that $q\left(z^{*}, l^{*}\right)=0$. Hence: $z^{*}=l^{*}$.

Corollary 3. Equip $(B, q)$ with an mw-distance mapping $p$. Assume $p$ is bounded with respect to the almost perfect function $\varphi$. Suppose that for all $e, l \in B$, we have:

$$
\varphi p\left(f_{1} e, f_{1} l\right) \leq k \max \left\{\varphi p\left(e, f_{1} e\right), \varphi p\left(l, f_{1} l\right)\right\}, \text { where } k \in[0,1) .
$$

Furthermore, assume that:

(i) $f$ is continuous;

or

(ii) if $u^{*} \in B$ and $u^{*} \neq f_{1} u^{*}$, then:

$$
\inf \left\{p\left(e, u^{*}\right)+p\left(f_{1} e, u^{*}\right): e \in B\right\}>0 .
$$

Then, $f_{1}$ has a unique fixed point in $B$.

Corollary 4. Equip $(B, q)$ with an mw-distance mapping $p$. Assume that there exists $A>0$ such that $p(e, l) \leq A$ for all $e, l \in B$. Furthermore, assume that for all $e, l \in B$, we have:

$$
p\left(f_{1} e, f_{1} l\right) \leq \alpha\left(p\left(e, f_{1} e\right)+p\left(l, f_{1} l\right)\right), \text { where } 0 \leq \alpha<\frac{1}{2} .
$$


Assume that:

(i) $f_{1}$ is continuous;

or

(ii) if $u^{*} \in B$ and $u^{*} \neq f_{1} u^{*}$, then:

$$
\inf \left\{p\left(e, u^{*}\right)+p\left(f_{1} e, u^{*}\right): e \in B\right\}>0 .
$$

Then, $f_{1}$ has a unique fixed point in $B$.

Proof. Define the almost perfect function $\varphi$ via $\varphi(e)=e$ in Corollary 3. Then:

$$
\begin{aligned}
\varphi\left(p\left(f_{1} e, f_{1} l\right)\right) & =p\left(f_{1} e, f_{1} l\right) \\
& \leq \lambda\left(p\left(e, f_{1} e\right)+p\left(l, f_{1} l\right)\right) \\
& \leq 2 \lambda \max \left\{p\left(e, f_{1} e\right), p\left(l, f_{1}\right)\right\} \\
& \left.=2 \lambda \max \left\{\varphi\left(p\left(e, f_{1} e\right)\right), \varphi\left(p\left(l, f_{1} l\right)\right)\right)\right\} .
\end{aligned}
$$

\section{Application}

In this section, we utilize Corollaries 1 and 4 to give some applications of our work.

Theorem 3. For any positive integer $n$, the equation:

$$
n x^{n}-x^{n-1}+4 n x-2=0
$$

has a unique solution in $[0,1]$.

Proof. Let $B=[0,1]$. Define $q: B \times B \rightarrow \mathbb{R}^{+}$by $q(x, y)=|x-y|$. Then, $(B, q)$ is a complete quasi metric space. Furthermore, define $p: B \times B \rightarrow[0, \infty)$ by $p(x, y)=|x-y|$. Then, $p$ is an $m \omega$-distance mapping. Now, equip $(B, q)$ with $p$.

Define $f_{1}: B \rightarrow B$ by:

$$
f_{1}(x)=\frac{x^{n-1}+2}{n\left(x^{n-1}+4\right)} .
$$

Furthermore, define $\varphi:[0, \infty) \rightarrow[0, \infty)$ by:

$$
\varphi(a)= \begin{cases}a^{2} & \text { if } a \in[0,1] \\ a^{2}+\frac{1}{2} & \text { if } a>1\end{cases}
$$

Note that $\varphi$ is an almost perfect function and $p$ is bounded with respect to $\varphi$. For $x, y \in B$, we have:

$$
\begin{aligned}
\varphi p\left(f_{1} x, f_{1} y\right) & =\frac{1}{n^{2}}\left|\frac{x^{n-1}+2}{x^{n-1}+4}-\frac{y^{n-1}+2}{y^{n-1}+4}\right|^{2} \\
& =\frac{1}{n^{2}}\left|\frac{2 x^{n-1}-2 y^{n-1}}{\left(x^{n-1}+4\right)\left(y^{n-1}+4\right)}\right|^{2} \\
& \leq \frac{4(n-1)^{2}}{n^{2}}\left(\frac{1}{\left(x^{2}+4\right)^{2}\left(y^{2}+4\right)^{2}}\right)|x-y|^{2} \\
& \leq \frac{(n-1)^{2}}{64 n^{2}}|x-y|^{2} \\
& =\frac{(n-1)^{2}}{64 n^{2}} \varphi p(x, y) .
\end{aligned}
$$


By taking $k=\frac{(n-1)^{2}}{64 n^{2}}$ and noting that $f_{1}$ is continuous, we conclude that $f_{1}$ satisfies all conditions of Corollary 1 . Thus, $f_{1}$ has a unique fixed point. Note that the unique fixed point of $f_{1}$ is the unique solution of:

$$
n x^{n}-x^{n-1}+4 n x-2=0 .
$$

Example 2. The equation:

$$
1000 x^{1000}-x^{999}+4000 x-2=0
$$

has a unique solution in $[0,1]$.

Proof. It follows from Theorem 3 by taking $n=1000$.

Let $Y$ be the set of non-decreasing functions $\tau: \mathbb{R}^{+} \rightarrow \mathbb{R}^{+}$such that $\tau$ is Lebesgue integrable for all compact sets in $\mathbb{R}^{+}$and:

$$
\int_{0}^{\mu} \tau(v) d v>0 \text { where } \mu>0
$$

Theorem 4. Equip $(B, q)$ with an mw-distance mapping $p$. Assume that there exists $A>0$ such that $p(e, l) \leq A$ for all $e, l \in B$. Furthermore, suppose the following condition:

(i) $f_{1}$ is continuous.

(ii) There exists $\tau \in \mathrm{Y}$ and $\alpha \in[0,1 / 2)$ such that for all $e, l \in B$, we have:

$$
\int_{0}^{p\left(f_{1} e, f_{1} l\right)} \tau(v) d v \leq \alpha\left(\int_{0}^{p\left(e, f_{1} e\right)} \tau(v) d v+\int_{0}^{p\left(l, f_{1} l\right)} \tau(v) d v\right)
$$

Then, $f_{1}$ has a unique fixed point in $B$.

Proof. Let $\varphi=\int_{0}^{t} \tau(v) d v$. Then, $\varphi$ is an almost perfect function. Corollary 4 ensures that $f_{1}$ has a unique fixed point in $B$.

\section{Conclusions}

The notions of $(\omega, \varphi)$-Suzuki contraction and generalized $(\omega, \varphi)$-Suzuki contraction are introduced. According to these nations many fixed point results are investigated. Some applications are introduced on the obtained results.

Author Contributions: Supervision: A.T. and W.S. Writing, original draft: T.Q., A.T., and W.S. Writing, review and editing: W.S.

Funding: This research is funded by Prince Sultan University through research group NAMAM, Group Number RG-DES-2017-01-17.

Acknowledgments: The authors would like to thank the anonymous reviewers and Editor for their valuable remarks on our paper. Furthermore, the third author thanks Prince Sultan University for funding this work through research group NAMAM, Group Number RG-DES-2017-01-17.

Conflicts of Interest: The authors declare no conflict of interest.

\section{References}

1. Banach, S. Sur les Opération dans les ensembles abstraits et leur application aux equations integral. Fundam. Math. 1922, 3, 133-181. [CrossRef]

2. Suzuki, T. A generalized Banach contraction principle that characterizes metric completeness. Proc. Am. Math. Soc. 2008, 136, 1861-1869. [CrossRef]

3. Kannan, R. Some results on fixed point. Bull. Calcutta Math. Soi. 1968, 60, 71-76. 
4. Wilson, W.A. On quasi-metric spaces. Am. J. Math. 1931, 53, 675-684. [CrossRef]

5. Aydi, H.; Jellali, M.; Karapınar, E. On fixed point results for $\alpha$-implicit contractions in quasi metric spaces and consequences. Nonlinear Anal. Model. Control 2016, 21, 40-56. [CrossRef]

6. Jleli, M.; Samet, B. Remarks on G-metric spaces and fixed point theorems. Fixed Point Theory Appl. 2012, 2012, 210. [CrossRef]

7. Bilgili, N.; Karapınar, E.; Samet, B. Eneralized $\alpha-\psi$ contractive mapping in quasi metric spaces and related fixed point theorems. J. Inequal. Appl. 2014, 2014, 36. [CrossRef]

8. Shatanawi, W.; Noorani, M.S.; Alsamir, H.; Bataihah, A. Fixed and common fixed point theorems in partially ordered quasi metric spaces. J. Math. Comput. Sci. 2012, 16, 516-528. [CrossRef]

9. Shatanawi, W.; Pitea, A. Some coupled fixed point theorems in quasi-partial metric spaces. Fixed Point Theory Appl. 2013, 2013, 2013:153. [CrossRef]

10. Alegre, C.; Marin, J. Modified $\omega$-distance on quasi metric spaces and fixed point theorems on complete quasi metric spaces. Topol. Appl. 2016, 203, 120-129. [CrossRef]

11. Nuseir, I.; Shatanawi, W.; Abu-Irwaq, I.; Batiahah, A. Nonlinear contraction and fixed point theorems with modified $\omega$-distance mappings in complete quasi metric spaces. J. Nonlinear Sci. Appl. 2017, 10, 5342-5350. [CrossRef]

12. Abodayeh, K.; Batiahah, A.; Shatanawi, W. Generalized $\Omega$-distance mappings and some fixed point theorems. Politehn. Univ. Bucharest Sci. Bull. Ser. A Appl. Math. Phys. 2017, 79, 223-232.

13. Mongkolkeha, C.; Cho, Y.J. Some coincidence point theorems in ordered metric spaces via $\omega$-distance. Carapthan J. 2018, 34, 207-214.

14. Abodayeh, K.; Shatanawi, W.; Bataihah, A.; Ansari, A.H. Some fixed point and common fixed point results through $\Omega$-distance under nonlinear contraction. Gazi University Journal of Science 2017, 30, $293-302$.

15. Khan, M.S.; Swaleh, M.; Sessa, S. Fixed point theorems by altering distances between the points. Bull. Aust. Math. Soc. 1984, 30, 1-9.

(C) 2019 by the authors. Licensee MDPI, Basel, Switzerland. This article is an open access article distributed under the terms and conditions of the Creative Commons Attribution (CC BY) license (http:/ / creativecommons.org/licenses/by/4.0/). 